\title{
Fillings, finite generation and direct limits of relatively hyperbolic groups
}

\author{
Daniel Groves and Jason Fox Manning*
}

\begin{abstract}
We examine the relationship between finitely and infinitely generated relatively hyperbolic groups. We observe that direct limits of relatively hyperbolic groups are in fact direct limits of finitely generated relatively hyperbolic groups. We combine this observation with known results to prove the Strong Novikov Conjecture for some exotic groups constructed by Osin.
\end{abstract}

Mathematics Subject Classification (2000). 20F67, 20F65, 46L89.

Keywords. Relatively hyperbolic groups, uniform embeddings, Novikov Conjecture.

\section{Introduction}

This paper is about the relationship between finitely generated and infinitely generated relatively hyperbolic groups. Most definitions and characterizations of relatively hyperbolic groups [8], [7], [3], [25], [6] assume finite generation at the outset. One exception is that of Osin [19]. In this definition, a group, finitely presented relative to a system of subgroups, is hyperbolic relative to that system if and only if the relative isoperimetric function is linear. (See Section 2 for definitions.)

Of interest to conjectures such as the Baum-Connes Conjecture and the Novikov Conjecture are 'exotic' groups, which may be used to test these conjectures. One way to build such exotic groups is by taking direct limits of hyperbolic groups, a method brought to great prominence by Olshanskii (see, for example, [15]). However, hyperbolic groups are known to satisfy the Baum-Connes Conjecture (this is due to Mineyev and $\mathrm{Yu}$ [13]), and the rational injectivity of the Baum-Connes assembly map is preserved by direct limits. This implies the Novikov Conjecture for groups built as direct limits of hyperbolic groups.

* The first author was supported in part by NSF Grant DMS-0504251. The second author was supported in part by an NSF Mathematical Sciences Postdoctoral Research Fellowship. Both authors thank the NSF for their support. We also thank Mark Sapir for asking us questions which led to the results in this paper, Guoliang Yu for help on the (Strong) Novikov Conjecture, and the referee for several useful comments. 
There are by now many results of the form: Suppose that $U$ is a property which implies the Novikov Conjecture (or the Strong Novikov Conjecture, or the Baum-Connes Conjecture), and suppose that $G$ is a (finitely generated) group which is hyperbolic relative to groups satisfying $\mathcal{U}$. Then $G$ satisfies $U$. (See, for example, [17], [5], [20]).

It appears then that finitely generated relatively hyperbolic groups are not a reasonable place to search for counterexamples to these conjectures, for likely one would have to build the pathology into the parabolic subgroups, and relative hyperbolicity would be irrelevant. In [16], Osin constructs groups with some remarkable properties as direct limits of infinitely generated relatively hyperbolic groups. One might think that these more flexible constructions might be of some help in building counterexamples to the Strong Novikov Conjecture. The main result of this paper (see Theorem 13) shows that this is probably not the case: Behavior exotic enough to defy the Strong Novikov Conjecture would have to be built into the finitely generated subgroups of the parabolic subgroups.

An outline of this paper is as follows: In Section 2 we introduce the notion of the finitely generated core of a relatively hyperbolic group (this concept is implicit in [19]). In Section 3 we explain how the main result in Osin's paper [18] about Dehn filling in relatively hyperbolic groups follows from the version where the relatively hyperbolic groups are assumed to be finitely generated. (In [10] the authors proved this finitely generated version under the additional assumption that the group is torsionfree.) In Section 4 we record some simple observations about direct limits of relatively hyperbolic groups. In particular, a direct limit of relatively hyperbolic groups is in fact the direct limit of finitely generated relatively hyperbolic groups. In Section 5 we use the main result of Section 4 (along with known results about uniform embeddability and the Strong Novikov Conjecture) to deduce the main result of this paper: that many of the remarkable groups constructed in [16] satisfy the Strong Novikov Conjecture (see Theorem 13).

\section{The finitely generated core}

Everything in this section is also contained in [19]; only the term finitely generated core is new.

Suppose that $G$ is a group with a family of subgroups $\left\{H_{\lambda}\right\}_{\lambda \in \Lambda}$, so that $G$ has a finite relative presentation

$$
G=\left\langle S,\left\{H_{\lambda}\right\}_{\lambda \in \Lambda} \mid \mathcal{R}\right\rangle,
$$

where $S$ is the finite relative generating set, and

$$
\mathcal{R} \subset \mathcal{F}(S) *\left(*_{\lambda \in \Lambda} H_{\lambda}\right)
$$

is a finite set of defining relations. Here $\mathcal{F}(S)$ denotes the free group on the letters $S$. We briefly recall the meaning of (1) from [19]. Let $\mathscr{H}=\bigcup_{\lambda \in \Lambda} H_{\lambda}$ be the disjoint 
union of the subgroups $\left\{H_{\lambda}\right\}$. For each $\lambda$, let $\mathcal{R}_{\lambda}$ be the collection of words in the alphabet $H_{\lambda}$ which represent the identity in $H_{\lambda}$. The relative presentation (1) is shorthand for the (non-relative) presentation

$$
G=\left\langle S \cup \mathcal{H} \mid \mathcal{R} \cup \mathcal{R}_{\Lambda}\right\rangle,
$$

where $\mathcal{R}_{\Lambda}=\bigcup_{\lambda \in \Lambda} \mathcal{R}_{\lambda}$. If $D$ is a van Kampen diagram over the presentation (2), then it has a relative area which is the number of 2-cells in the diagram labelled by elements of $\mathcal{R}$. Let $w$ be a word in the alphabet $S \cup \mathscr{H}$ which represents the identity in $G$. The relative area of $w$ is the smallest relative area of any van Kampen diagram over (2) whose boundary is labelled by $w$.

Definition 1. A group with finite relative presentation $G=\left\langle S,\left\{H_{\lambda}\right\}_{\lambda \in \Lambda} \mid \mathcal{R}\right\rangle$ is hyperbolic relative to the collection of subgroups $\left\{H_{\lambda}\right\}_{\lambda \in \Lambda}$ if the relative isoperimetric function is linear, in the following sense: There is a constant $C$ so that every word of length $n$ in the alphabet $S \cup \mathscr{H}$ which represents the identity in $G$ can be filled by a van Kampen diagram of relative area at most $C n$.

Given a relative presentation as in (1), each $r \in \mathcal{R}$ can be written in normal form as some product

$$
r=w_{1} h_{1} \ldots w_{n} h_{n}
$$

with each $w_{i}$ a word in the free group $\mathcal{F}(S)$ and each $h_{i}$ in $H_{\lambda_{i}}$ for some $\lambda_{i} \in \Lambda$. Let $\Omega$ be the set of nontrivial elements of $\bigcup_{\lambda} H_{\lambda}$ which occur in one of these normal forms. Since $\Omega$ is finite, the set $I=\left\{\lambda \in \Lambda \mid H_{\lambda} \cap \Omega \neq \emptyset\right\}$ is also finite. For each $i \in I, \Omega \cap H_{i}$ (finitely) generates some $H_{i}^{\prime}<H_{i}$. Let $G^{\prime}$ be the subgroup of $G$ generated by $S \cup\left(\bigcup_{i \in I} H_{i}^{\prime}\right)$. We call $G^{\prime}$ the finitely generated core of $G$ associated to the presentation $\left\langle S,\left\{H_{\lambda}\right\}_{\lambda \in \Lambda} \mid \mathcal{R}\right\rangle$. (The finitely generated core is highly nonunique; see Remark 5.) The next three lemmas are contained in the statement and proof of Theorem 2.44 in [19]. (In Osin's statement, the finitely generated core is called $Q$.) The second is straightforward; the first and third can be proved using simple variations on the arguments in Section 3.

Lemma 2. If $G^{\prime}$ is the finitely generated core of $G$ coming from the finite relative presentation $\left\langle S,\left\{H_{\lambda}\right\}_{\lambda \in \Lambda} \mid \mathcal{R}\right\rangle$, then $G^{\prime}$ has a finite relative presentation

$$
G^{\prime}=\left\langle S,\left\{H_{i}^{\prime}\right\}_{i \in I} \mid \mathcal{R}\right\rangle
$$

(Note that

$$
\mathcal{R} \subset \mathcal{F}(S) *\left(*_{i \in I} H_{i}^{\prime}\right) \subset \mathcal{F}(S) *\left(*_{\lambda \in \Lambda} H_{\lambda}\right),
$$

so the presentation in (3) is at least well defined.) 
Lemma 3. If $G$ is given by the finite relative presentation $\left\langle S,\left\{H_{\lambda}\right\} \lambda \in \Lambda \mid \mathcal{R}\right\rangle$, and $G^{\prime}=\left\langle S,\left\{H_{i}^{\prime}\right\}_{i \in I} \mid \mathcal{R}\right\rangle$ is the corresponding finitely generated core of $G$, as in the first paragraph, then

$$
G=G_{0} *\left(* \lambda \in \Lambda \backslash I H_{\lambda}\right)
$$

where $G_{0}$ is the subgroup of $G$ generated by $S \cup\left(\bigcup_{i \in I} H_{i}\right)$.

Lemma 4. $G=\left\langle S,\left\{H_{\lambda}\right\}_{\lambda \in \Lambda} \mid \mathcal{R}\right\rangle$ is hyperbolic relative to $\left\{H_{\lambda}\right\}_{\lambda \in \Lambda}$ if and only if its finitely generated core $G^{\prime}$ is hyperbolic relative to $\left\{H_{i}^{\prime}\right\}_{i \in I}$.

Remark 5. If $G$ has a finite relative presentation

$$
G=\left\langle S,\left\{H_{\lambda}\right\} \lambda \in \Lambda \mid \mathcal{R}\right\rangle
$$

and $T \subseteq G$ is finite, then there is obviously a finite relative presentation

$$
G=\left\langle S \cup T,\left\{H_{\lambda}\right\} \lambda \in \Lambda \mid \mathcal{R}^{\prime}\right\rangle
$$

for some (finite) set of relators $\mathcal{R}^{\prime}$ containing $\mathcal{R}$. Thus given the finite set $T \subseteq G$, there is always a finitely generated core containing $T$. This observation will be used in Section 4.

\section{Fillings and cores}

In [18], Osin proved the following theorem:

Theorem 6 ([18], Theorem 1.1). Suppose that $G$ is hyperbolic relative to the system of subgroups $\left\{H_{\lambda}\right\}_{\lambda \in \Lambda}$. Then there is a finite set $B \subset \bigcup_{\lambda} H_{\lambda}$ so that if $\left\{K_{\lambda}\right\}_{\lambda \in \Lambda}$ is a collection of subgroups so that for each $\lambda \in \Lambda$, we have

(1) $K_{\lambda} \triangleleft H_{\lambda}$, and

(2) $K_{\lambda} \cap B=\emptyset$,

then

(1) the natural map $\phi_{\lambda}: H_{\lambda} / K_{\lambda} \rightarrow G /\left\langle\left\langle\bigcup_{\lambda} K_{\lambda}\right\rangle\right.$ is an injection, and

(2) $G /\left\langle\left\langle\bigcup_{\lambda} K_{\lambda}\right\rangle\right.$ is hyperbolic relative to $\left\{\phi_{\lambda}\left(H_{\lambda} / K_{\lambda}\right)\right\}_{\lambda \in \Lambda}$.

(Here $\langle\langle S\rangle$ is the normal subgroup generated by the set $S$.)

In [10], we proved the same theorem, with the additional assumptions that $G$ is torsion-free and that the (finitely many) parabolic subgroups are finitely generated.

The torsion-free assumption in [10] is technical, and will not be addressed here. However, we remarked in [10], Remark 1.5, that if one can prove Theorem 6 under the additional assumptions that $\Lambda$ is finite and each $H_{\lambda}$ is finitely generated, then the full statement follows. This is the content of this section. 
Proposition 7. If Theorem 6 holds with the additional hypotheses that $\Lambda$ is finite and that $H_{\lambda}$ is finitely generated for each $\lambda \in \Lambda$, then Theorem 6 holds in full generality.

Proof. Let $G=\left\langle S,\left\{H_{\lambda}\right\}_{\lambda \in \Lambda} \mid \mathcal{R}\right\rangle$ and $G^{\prime}$ the finitely generated core of $G$ relative to $\left\langle S,\left\{H_{\lambda}\right\}_{\lambda \in \Lambda} \mid \mathcal{R}\right\rangle$. By Lemma $2,\left\langle S,\left\{H_{i}^{\prime}\right\}_{i \in I} \mid \mathcal{R}\right\rangle$ is a relative presentation for $G^{\prime}$. Lemma 3 implies that $G$ splits as a free product

$$
G=G_{0} *\left(* \lambda \in \Lambda \backslash I H_{\lambda}\right)
$$

where $G_{0}$ is the subgroup of $G$ generated by $S \cup\left(\bigcup_{i \in I} H_{i}\right)$. Arbitrarily filling peripheral subgroups $H_{\lambda}$ for $\lambda \notin I$ does not affect the free product structure of $G$ (or relative hyperbolicity). We may therefore assume that $\Lambda=I$, and so $G=G_{0}$.

Lemma 4 implies that $G^{\prime}$ is hyperbolic relative to $\left\{H_{i}^{\prime}\right\}_{i \in I}$, so we may apply the finitely generated version of Theorem 6 to $G^{\prime}$. Let $B$ be the finite subset of $\bigcup_{\lambda} H_{\lambda}^{\prime}$ of "forbidden" elements for peripheral fillings of $G^{\prime}$ coming from Theorem 6 in the finitely generated case. We show that $B$ also suffices as the set of forbidden elements for $G$.

For each $i \in I$, let $K_{i} \triangleleft H_{i}$ satisfy $K_{i} \cap B=\emptyset$. The following is obvious:

Claim 7.1. If $K_{i}^{\prime}=K_{i} \cap H_{i}^{\prime}$, then $K_{i}^{\prime} \triangleleft H_{i}^{\prime}$ and $K_{i}^{\prime} \cap B=\emptyset$.

Let $N$ be the normal closure of $\bigcup_{i} K_{i}$ in $G$, and let $N^{\prime}$ be the normal closure of $\bigcup_{i} K_{i}^{\prime}$ in $G^{\prime}$. Write $\bar{H}_{i}$ for the image of $H_{i}$ under the quotient map $G \rightarrow G / N$, and write $\bar{H}_{i}^{\prime}$ for the image of $H_{i}^{\prime}$ under the quotient map $G^{\prime} \rightarrow G^{\prime} / N^{\prime}$. Theorem 6 in the finitely generated case and Claim 7.1 together imply that $G^{\prime} / N^{\prime}$ is hyperbolic relative to $\left\{\bar{H}_{i}^{\prime}\right\}_{i \in I}$.

We will show that $G^{\prime} / N^{\prime}$ is a finitely generated core of $G / N$; Lemma 4 and Claim 7.3 (below) then imply the proposition.

We introduce some notation in this paragraph. Let $\mathscr{H}$ be the disjoint union of the $H_{i}$ for $i \in I$ and let $\mathcal{R}_{I}$ be the collection of words representing the identity in some $H_{i}$. Finally, let $\mathcal{K}$ be the disjoint union of the $K_{i}$ for $i \in I$. The quotient group $G / N$ has the presentation

$$
G / N=\left\langle S \cup \mathscr{H} \mid \mathcal{R} \cup \mathcal{R}_{I} \cup \mathcal{K}\right\rangle
$$

which can also be written as the relative presentation

$$
G / N=\left\langle S,\left\{H_{i}\right\}_{i \in I} \mid \mathcal{R} \cup \mathcal{K}\right\rangle .
$$

Let $w \in \mathscr{F}(S) *\left(*_{i \in I} H_{i}\right)$ be a word which is trivial in $G / N$. There is a van Kampen diagram over (4) for $w$ containing five possible kinds of two-cells:

(1) $\mathcal{R}$-cells,

(2) $H^{\prime}$-cells: cells representing relations in $H_{i}^{\prime}$ for some $i \in I$, 
(3) $K^{\prime}$-cells: cells representing elements of $K_{i}^{\prime}<G$ for some $i \in I$,

(4) $H \backslash H^{\prime}$-cells: cells representing relations in $H_{i}$ for some $i \in I$ which involve some elements of $H_{i} \backslash H_{i}^{\prime}$, and

(5) $K \backslash K^{\prime}$-cells: cells representing elements of $K_{i} \backslash K_{i}^{\prime}$ for some $i \in I$.

The first three kinds of 2-cells will be called good, the last two bad. A bad patch $P$ is a maximal union of bad 2-cells, subject to the condition that the interior of $P$ is connected.

Each edge of the van Kampen diagram is labelled by some element of $S \cup\left(\bigcup_{i} H_{i} \backslash\{1\}\right)$. Edges of the van Kampen diagram will be called good if they are labelled by elements of $S \cup\left(\bigcup_{i} H_{i}^{\prime}\right)$; otherwise they are bad. Every bad 2-cell has at least one bad edge in its boundary, whereas good 2-cells have no bad edges in their boundary.

The point of the good/bad notation is that if we can modify a van Kampen diagram so that it contains only good 2-cells, then it follows that its boundary represents the trivial element of $G^{\prime} / N^{\prime}$.

Claim 7.2. For each $i \in I$, the natural map from $H_{i}^{\prime} / K_{i}^{\prime}$ to $G / N$ is injective.

Proof. Let $w \in H_{i}^{\prime}$ be in the kernel of the map to $G / N$, and let $D$ be a van Kampen diagram for $w$ as described above. (In particular $\partial D$ consists of a single good edge.) If this van Kampen diagram can be modified to contain only good 2-cells, then $w$ is in the kernel of the natural map from $H_{i}^{\prime}$ to $G^{\prime} / N^{\prime}$, and thus by the finitely generated version of Theorem 6 we have $w \in K_{i}^{\prime}$.

Let $P$ be any bad patch. Note that all the 2-cells in $P$ have boundary labels in a single subgroup $H_{j}$, for some $j \in I$ which may be different from $i$.

Subclaim 7.2.1. Each component of $\partial P$ represents an element of $H_{j}^{\prime}$.

Proof. If not, then there is some bad edge in $\partial P$. This edge can only be adjacent to another bad 2-cell (in which case $P$ is not maximal) or to the boundary of the van Kampen diagram itself. Since the boundary of the van Kampen diagram is labelled only by good edges, we derive a contradiction.

We now suppose $P$ is a bad patch which is innermost in the following sense: No other bad patch is separated from the boundary of the van Kampen diagram by the interior of $P$. It is evident that if there are any bad patches, then at least one is innermost. Let $c_{P}$ be the outermost boundary of $P$.

We claim that the sub-diagram bounded by $c_{P}$ can be modified so that every 2-cell has all its boundary labels in $H_{j}$. If $P$ is simply connected, this is immediate. Suppose then that the innermost bad patch $P$ is not simply connected. Some component $c$ of $\partial P$ bounds a disk $D$ containing only good 2 -cells, since $P$ is innermost. Moreover, $c$ 
consists only of good edges. Reading the labels of the edges of $c$ gives some word $w_{c}$ in the alphabet $S \cup\left(\bigcup_{i} H_{i}^{\prime}\right)$, and the disk $D$ is itself a van Kampen diagram for $w_{c}$ in $G^{\prime} / N^{\prime}$. By Subclaim 7.2.1, $w_{c}$ represents some element of $H_{j}^{\prime}<G^{\prime}$. The disk $D$ is a demonstration that it represents the trivial element of $G^{\prime} / N^{\prime}$. Since $H_{j}^{\prime} / N_{j}^{\prime}$ injects into $G^{\prime} / N^{\prime}$ by assumption, it follows that $w_{c}$ represents an element of $N^{\prime}$. The disk $D$ may therefore be replaced by a single $K^{\prime}$-cell. Applying this argument in turn to each inner boundary component of $P$, we fill $c_{P}$ entirely by 2-cells with boundary labels in $H_{j}$.

Let $w_{P}$ be the word in $S \cup\left(\bigcup_{i} H_{i}^{\prime}\right)$ given by the labels of $c_{P}$. By the previous paragraph, $w_{P}$ represents the trivial element of $H_{j} / K_{j}$. Moreover, by Subclaim 7.2.1, it lies in $H_{j}^{\prime}$. Since $H_{j}^{\prime} / K_{j}^{\prime}$ injects into $H_{j} / K_{j}, w_{P}$ represents an element of $K_{j}^{\prime}$. We therefore can replace the bad patch $P$ (and any disks attached to its interior) with a single $K^{\prime}$-cell, thus reducing the number of bad cells in the van Kampen diagram. Iterating this procedure, all bad cells can be removed, and Claim 7.2 follows.

Claim 7.3. For each $i \in I$, the natural map from $H_{i} / K_{i}$ to $G / N$ is injective.

Proof. We now assume that $w \in H_{i} \backslash H_{i}^{\prime}$ and suppose that $w$ lies in $N$. Again we can build a van Kampen diagram $D$ for $w$, this time with boundary equal to a single bad edge. We argue as in Claim 7.2: First, there is a single outermost bad patch (since $\partial D$ is a single bad edge), and the boundary of each other bad patch is an element of some $H_{j}^{\prime}$. We now reduce the number of bad patches until $D$ contains a single bad patch and no $\mathcal{R}$-cells. It follows that $w$ is already trivial in $H_{i} / K_{i}$, and Claim 7.3 is proved.

We now turn to the proof of Proposition 7. By Claim 7.3, we can regard each $H_{i} / K_{i}$ as a subgroup of $G / N$. The subgroup $N$ of $G$ is the normal closure of the set $\bigcup K_{i}$, so we obtain a finite relative presentation

$$
G / N=\left\langle S,\left\{H_{i} / K_{i}\right\}_{i \in I} \mid \overline{\mathcal{R}}\right\rangle,
$$

where $\overline{\mathcal{R}}$ is the image of $\mathcal{R}$ in $\mathcal{F}(S) *\left(*_{i \in I} H_{i} / K_{i}\right)$ under the obvious map from $\mathcal{F}(S) *\left(*_{i \in I} H_{i}\right)$. It remains to observe that (regarding $H_{i}^{\prime} / K_{i}^{\prime}$ as a subgroup of $H_{i} / K_{i}$ ) the group $G^{\prime} / N^{\prime}$ is the finitely generated core coming from the presentation (5),

$$
G^{\prime} / N^{\prime}=\left\langle S,\left\{H_{i}^{\prime} / K_{i}^{\prime}\right\}_{i \in I} \mid \overline{\mathcal{R}}\right\rangle .
$$

This completes the proof of Proposition 7.

\section{Direct limits of relatively hyperbolic groups}

Direct limits of groups are a particularly good way of building finitely generated groups with interesting properties. This idea was developed by Olshanskii [15] with direct 
limits of hyperbolic groups, and recently by Osin [16] for direct limits of relatively hyperbolic groups. We are interested in the relatively hyperbolic construction.

Definition 8. Suppose that $\left\{G_{i}\right\}_{i \in \mathbb{N}}$ is a sequence of groups, and $\left\{\phi_{i}: G_{i} \rightarrow G_{i+1}\right\}$ is a sequence of homomorphisms.

Let $X=\prod_{i \in \mathbb{N}} G_{i}$ be the Cartesian product of the $G_{i}$. Define a subset $\Lambda \subset X$ as follows:

$$
\Lambda=\left\{\left(g_{i}\right) \mid \text { there exists } J \text { such that for all } j \geq J, g_{j+1}=\phi_{j}\left(g_{j}\right)\right\} .
$$

Put an equivalence relation ' $\sim$ ' on $\Lambda$ so that $\left(g_{i}\right)=\left(h_{i}\right)$ if there is some $K$ so that for all $k \geq K$ we have $g_{k}=h_{k}$.

Let $L=\Lambda / \sim$. The group operation on $X$ descends to $L$, and $L$ is a group, called the direct limit of $\left\{\left(G_{i}, \phi_{i}\right)\right\}$.

Remark 9. Using the definition above, if the groups $G_{i}$ are countable then so is the limit $L$. If one were to allow more general directed systems then this would no longer be the case. However, in order to understand the constructions from [16], the above definition is sufficient.

We will need the following lemma.

Lemma 10. Suppose that $G$ is a countable relatively hyperbolic group. Then the collection of (nontrivial) parabolic subgroups of $G$ is countable.

Proof. Let $\Lambda$ be the index set for the parabolic subgroups $\left\{H_{\lambda}\right\}_{\lambda \in \Lambda}$ of $G$. We suppose that each $H_{\lambda}$ is nontrivial. We have to show that $\Lambda$ is countable.

Let $G^{\prime}$ be a finitely generated core of $G$, and suppose that $G^{\prime}$ is hyperbolic relative to $\left\{H_{i}^{\prime}\right\}_{i \in I}$.

By Lemma 3 we have

$$
G=G_{0} *\left(* \lambda \in \Lambda \backslash I H_{\lambda}\right),
$$

where $G_{0}$ is hyperbolic relative to $\left\{H_{i}\right\}_{i \in I}$. A countable group cannot contain uncountably many nontrivial free factors, so $\Lambda \backslash I$ is countable, which implies that $\Lambda$ is countable, as required.

Proposition 11. Suppose that $G$ is a group which is isomorphic to a direct limit of groups $\left\{G_{i} \stackrel{\phi_{i}}{\longrightarrow} G_{i+1} \mid i \in \mathbb{N}\right\}$ so that each $G_{i}$ is countable and hyperbolic relative to some collection of proper subgroups $\mathcal{P}_{i}$. Then for every $i$, there exists a finite relative presentation $\operatorname{Pres}_{i}$ of $G_{i}$ with respect to $\mathcal{P}_{i}$ and homomorphisms $\phi_{i}^{\prime}: G_{i}^{\prime} \rightarrow G_{i+1}^{\prime}$ such that $G$ is isomorphic to the direct limit of finitely generated groups $\left\{G_{i}^{\prime} \stackrel{\phi_{i}^{\prime}}{\longrightarrow} G_{i+1}^{\prime} \mid i \in \mathbb{N}\right\}$, where $G_{i}^{\prime}$ is the finitely generated core of $G_{i}$ associated to Pres $_{i}$ for each $i$. 
Proof. Suppose that $G$ is the direct limit of $\left\{\left(G_{i}, \phi_{i}\right)\right\}$ where $\phi_{i}: G_{i} \rightarrow G_{i+1}$, and the $G_{i}$ are all relatively hyperbolic.

Let $\mathcal{P}_{i}=\left\{P_{1}^{i}, P_{2}^{i}, \ldots\right\}$ be the parabolic subgroups of $G_{i}$, and $X_{i}$ a finite relative generating set for $G_{i}$ with respect to $\mathcal{P}_{i}$.

The group $G$ is countable, so let $\left\{g_{0}, g_{1}, g_{2}, \ldots\right\}$ be an enumeration of its elements. For each $i \geq 0$, let $j(i)$ be the least number so that (i) $j(i) \geq j(i-1)+1$; and (ii) the image of $G_{j(i)}$ in $G$ contains $\left\{g_{0}, \ldots, g_{i}\right\}$. Since we may pass to a subsequence without changing the limit, for ease of notation we will suppose that $j(i)=i$.

Let $Y_{i}=\left\{g_{0}^{i}, \ldots, g_{i}^{i}\right\}$ be a subset of $G_{i}$ so that the map from $G_{i}$ to $G$ sends $g_{l}^{i}$ to $g_{l}$.

We will define a collection of finitely generated subgroups $G_{i}^{\prime}$ of $G_{i}$, and homomorphisms $\phi_{i}^{\prime}: G_{i}^{\prime} \rightarrow G_{i+1}^{\prime}$ so that (i) the direct limit of $\left\{\left(G_{i}^{\prime}, \phi_{i}^{\prime}\right)\right\}$ is $G$; and (ii) each $G_{i}^{\prime}$ is relatively hyperbolic. In fact, the map $\phi_{i}^{\prime}$ will be the restriction of $\phi_{i}$ to $G_{i}^{\prime}$, and we will use the notation $\phi_{i}$ for this map also.

Define $G_{0}^{\prime}$ to be the finitely generated core of $G_{0}$ with respect to a finite relative presentation with the relative generating set $Z_{0}=X_{0} \cup Y_{0}$.

Suppose that, for $r<i$, we have defined $G_{r}^{\prime}$ (with finite relative generating set $Z_{r}$, and finitely many finitely generated parabolics) and $\phi_{r-1}^{\prime}$. We define $G_{i}^{\prime}$ as follows:

Let $W_{i}=\phi_{i-1}\left(Z_{i-1}\right) \in G_{i}$, and let $Z_{i}=W_{i} \cup X_{i} \cup Y_{i}$. The set $Z_{i}$ is a finite relative generating set for $G_{i}$, so there is some finite relative presentation Pres ${ }_{i}=$ $\left\langle Z_{i}, \mathcal{P}_{i} \mid \mathcal{R}_{i}\right\rangle$. Define $G_{i}^{\prime}$ to be the finitely generated core of $G_{i}$ associated to $\operatorname{Pres}_{i}$.

By Lemma 4, the group $G_{i}^{\prime}$ is hyperbolic relative to its finitely many nontrivial intersections with the elements of $\mathcal{P}_{i}$, and these intersections are themselves finitely generated.

We claim that the direct limit of $\left\{\left(G_{i}^{\prime}, \phi_{i}\right)\right\}$ is isomorphic to $G$. Let $L$ be the direct limit of $\left\{\left(G_{i}^{\prime}, \phi_{i}\right)\right\}$. Since each $G_{i}^{\prime}$ is a subgroup of $G_{i}$, and the map $\phi_{i}: G_{i}^{\prime} \rightarrow G_{i+1}^{\prime}$ is a restriction of the homomorphism $\phi_{i}: G_{i} \rightarrow G_{i+1}$, there is an obvious map $\pi: L \rightarrow G$. We construct the inverse map $\pi^{-1}: G \rightarrow L$ as follows: suppose that $g \in G$. Then there is some $i$ so that $g=g_{i}$. Then the choice of $j(i)$ (and the renumbering above) implies that for all $j \geq i$ there is an element $g_{i}^{j} \in G_{j}$ so that $g_{i}^{j}$ maps to $g$ under the canonical map from $G_{j}$ to $G$. But then $g_{i}^{j} \in G_{j}^{\prime}$ and $\phi_{j}\left(g_{i}^{j}\right)=g_{i}^{j+1}$. We set $\pi^{-1}(g)$ to be the sequence $\left(1, \ldots, 1, g_{i}^{i}, g_{i}^{i+1}, \ldots\right)$, where the first $i-1$ terms of this sequence are the identity element. It is clear that $\pi^{-1}$ is the inverse of $\pi$, and we have proved the proposition.

\section{Some examples of Osin and the (Strong) Novikov Conjecture}

In [16], Osin gives constructions of groups satisfying some remarkable properties. We show that if the input to these constructions is a uniformly embeddable group 
then the Strong Novikov Conjecture ${ }^{1}$ holds for the output. In particular were these constructions to yield a counterexample to the Strong Novikov Conjecture, then the input group must already have been rather exotic.

Uniform embeddability for groups was introduced by Gromov in [9]. However, it is now more common to use the following more general notion:

Definition 12. Let $\left(X, d_{1}\right)$ and $\left(Y, d_{2}\right)$ be metric spaces. A map $i: X \rightarrow Y$ is a uniform embedding if there are unbounded increasing functions $\rho_{1}, \rho_{2}: \mathbb{R}^{+} \rightarrow R^{+}$ so that for all $x, x^{\prime} \in X$,

$$
\rho_{1}\left(d_{1}\left(x, x^{\prime}\right)\right) \leq d_{2}\left(i(x), i\left(x^{\prime}\right)\right) \leq \rho_{2}\left(d_{1}\left(x, x^{\prime}\right)\right) .
$$

A countable group $G$ is called uniformly embeddable if there is a uniform embedding of $G$ into a Hilbert space.

In the terminology of Roe [21], Chapter 11, a group is uniformly embeddable if it admits a coarse embedding into Hilbert space. The connection between uniform embeddability and the Novikov Conjecture was established in [26], where the Novikov Conjecture was proved for a uniformly embeddable group whose classifying space has the homotopy type of a finite CW complex. The finiteness assumption is removed in [24], where the Strong Novikov Conjecture is established for all uniformly embeddable groups.

In the statement below, $\pi(K)$ is the set of (finite) orders of elements of a group $K$. A group $G$ is said to be verbally complete if the equation $w\left(x_{1}, \ldots, x_{n}\right)=g$ has a solution in $G$, for any $g \in G$, and for $w$ any (freely reduced, nonempty) word in any number of free variables. (In particular, every element of such a group is a commutator, has roots of all orders, and so on.)

Theorem 13. Given any uniformly embeddable countable group $G$, there exist 2-generated groups $\mathrm{H}_{1}$ and $\mathrm{H}_{2}$ satisfying:

(1) $G$ embeds in $H_{1}$ and in $H_{2}$.

(2) $H_{1}$ and $H_{2}$ satisfy the Strong Novikov Conjecture.

(3) $\pi\left(H_{1}\right)=\pi(G)$ and any two elements of $H_{1}$ with the same order are conjugate.

(4) $\mathrm{H}_{2}$ is verbally complete; moreover, if $G$ is torsion-free, then so is $\mathrm{H}_{2}$.

Before giving the proof of Theorem 13, we should remark that the construction (and most of the theorem) is due to Denis Osin in [16]; the sole innovation here is that point (2) can be guaranteed.

If we start with an infinite torsion-free uniformly embeddable group (like $\mathbb{Z}$ ), then Theorem 13 yields:

\footnotetext{
${ }^{1}$ by which we mean that the Baum-Connes assembly map is injective (see [1], [27])
} 
Corollary 14. There exists a 2-generated, infinite, torsion-free group $G$ which has two conjugacy classes and satisfies the Strong Novikov Conjecture.

Corollary 15. There exists a 2-generated, infinite, torsion-free group $G$ which is verbally complete and satisfies the Strong Novikov Conjecture.

The following proposition is used in the proof of Theorem 13.

Proposition 16. If $G$ is a uniformly embeddable group then $G$ can be embedded into countable groups $R_{1}$ and $R_{2}$ so that:

(1) Finitely generated subgroups of $R_{1}$ and $R_{2}$ are uniformly embeddable.

(2) $\pi\left(R_{1}\right)=\pi(G)$ and all elements of the same order are conjugate.

(3) $R_{2}$ is verbally complete.

Proof. For $R_{1}$ satisfying (2), we use a construction of Higman, Neumann, and Neumann [11] (see Lyndon and Schupp [12], Theorem IV.3.3). Let $G_{0}=G$. Suppose $G_{i-1}$ has been defined, and let $\left\{\left(\alpha_{j}, \beta_{j}\right) \mid j \in \mathbb{N}\right\}$ be the set of pairs of elements in $G_{i-1}$ so that the orders of $\alpha_{j}$ and $\beta_{j}$ are equal. Define $G_{i}$ by the presentation:

$$
G_{i}=\left\langle G_{i-1},\left\{t_{i, j}\right\}_{j \in \mathbb{N}} \mid t_{i, j}^{-1} \alpha_{j} t_{i, j}=\beta_{j}, j \in \mathbb{N}\right\rangle,
$$

and define $R_{1}=\bigcup_{i \in \mathbb{N}} G_{i}$ to be the direct limit of these groups.

For $R_{2}$ satisfying (3), we refer to the construction in [16]. We will use only the following facts:

- The group $R_{2}$ is also a union of subgroups $U_{i}$ for $i \in \mathbb{Z}_{\geq 0}$, and $U_{0}=G$.

- If $i \geq 1$, then $U_{i+1}$ is an amalgamated free product of $U_{i}$ with infinitely many groups $\left\{F_{j}^{i}\right\}_{j \in \mathbb{N}}$, where each $F_{j}^{i}$ is either a free group or a one-relator group with torsion, and each amalgamating subgroup is cyclic.

It remains only to prove that finitely generated subgroups of $R_{1}$ and $R_{2}$ are uniformly embeddable. We prove both simultaneously. Let $H$ be a finitely generated subgroup of $R_{1}$ or $R_{2}$. If $H<R_{1}$, then we will set $V_{i}=G_{i}$ for each $i$; if $H<R_{2}$, we set $V_{i}=U_{i}$. The group $H$ is contained in $V_{i}$ for some $i \in \mathbb{N}$. If $i=0$, we are done, since subgroups of uniformly embeddable groups are clearly uniformly embeddable. We may suppose by induction that finitely generated subgroups of $V_{i-1}$ are uniformly embeddable. Free groups and one relator groups with torsion are word hyperbolic ([14]; see [12], Theorem IV.5.5); their finitely generated subgroups therefore have finite asymptotic dimension (this is a result of Gromov; see [22]), and are therefore uniformly embeddable (see [21], Chapter 11). Thus $V_{i}$ is a graph of groups with cyclic edge groups and uniformly embeddable vertex groups. The group $H$ inherits a graph of groups decomposition from $V_{i}$; the edge groups are again cyclic. Since $H$ is finitely generated, the graph of groups decomposition of $H$ has a finite underlying 
graph. Also, since $H$ is finitely generated and the edge groups of the (finite) graph of groups are finitely generated, the vertex groups of the graph of groups are finitely generated. Thus by induction the vertex groups are uniformly embeddable. Now we may apply a theorem of Dadarlat and Guentner [4] to deduce that $H$ is uniformly embeddable.

Remark 17. Note that the proposition implies that $R_{1}$ and $R_{2}$ are uniformly embeddable, since countable locally uniformly embeddable groups are uniformly embeddable by [4].

The hypothesis and conclusion of uniform embeddability in Proposition 16 may be strengthened to finite asymptotic dimension, by applying theorems of Osin [17] and Bell and Dranishnikov [2] in place of the theorems of Dadarlat and Guentner quoted above.

Proof of Theorem 13. We do not describe Osin's constructions here, but refer the reader to [16], particularly to the overview in Section 2 of his paper.

The groups $H_{1}$ and $H_{2}$ are built as direct limits of (infinitely generated) relatively hyperbolic groups, with peripheral subgroup $R_{1}$ or $R_{2}$, respectively. At each stage the term in the direct limit is hyperbolic relative to $R_{1}$ or $R_{2}$ from the above proposition. The finitely generated cores of these terms are therefore hyperbolic relative to finitely generated subgroups of $R_{1}$ or $R_{2}$; these finitely generated subgroups are uniformly embeddable by Proposition 16. The cores are thus hyperbolic relative to uniformly embeddable subgroups; by the main result of [5] they are themselves uniformly embeddable. Thus, if $\Gamma$ is such a finitely generated core, then by [24, Theorem 6.1], the Baum-Connes assembly map with coefficients is injective (with any separable $\Gamma$ $C^{*}$-algebra coefficients). In particular, the Baum-Connes assembly map (with trivial coefficients) is injective for each such $\Gamma$, i.e. the finitely generated core $\Gamma$ satisfies the Strong Novikov Conjecture.

We have exhibited $H_{1}$ and $H_{2}$ as direct limits of groups satisfying the Strong Novikov Conjecture. By [23, Proposition 2.4], the Strong Novikov Conjecture is stable under taking direct limits, so $H_{1}$ and $H_{2}$ themselves satisfy the Strong Novikov Conjecture.

By the main result of [4], the class of countable groups which are uniformly embeddable is closed under direct limits of groups (where all of the maps are injective). It is clear from Lemma 4 and Remark 5 that if $G$ is a countable relatively hyperbolic group with parabolic subgroups $\left\{H_{i}\right\}_{i \in I}$ then $G$ is the direct limit of an increasing collection of finitely generated cores of $G$, each of which is hyperbolic relative to a collection of finitely generated subgroups of finitely many of the $H_{i}$. Therefore, we have the following generalization of the main result of [5].

Proposition 18. Suppose that $G$ is a (countable but not necessarily finitely generated) group which is hyperbolic relative to a (not necessarily finite) collection of subgroups 
$\left\{H_{i}\right\}_{i \in I}$, and suppose that each of the $H_{i}$ is uniformly embeddable. Then $G$ is uniformly embeddable.

Remark 19. We feel that it is worth remarking that although Proposition 11 proves that in theory Osin could have built his examples without using infinitely generated relatively hyperbolic groups, it is very difficult to see how to do this directly, and in any case infinitely generated relatively hyperbolic groups certainly make the proof conceptually easier.

\section{References}

[1] P. Baum, A. Connes, and N. Higson, Classifying space for proper actions and $K$-theory of group $C^{*}$-algebras. In $C^{*}$-algebras: 1943-1993 (San Antonio, TX, 1993), Contemp. Math. 167, Amer. Math. Soc., Providence, RI, 1994, 240-291. Zbl 0830.46061 MR 1292018

[2] G. Bell and A. Dranishnikov, On asymptotic dimension of groups acting on trees. Geom. Dedicata 103 (2004), 89-101. Zbl 02069061 MR 2034954

[3] B. Bowditch, Relatively hyperbolic groups. Preprint 1999. www.maths.soton.ac.uk/staff/Bowditch/preprints.html

[4] M. Dadarlat and E. Guentner, Constructions preserving Hilbert space uniform embeddability of discrete groups. Trans. Amer. Math. Soc. 355 (2003), 3253-3275. Zbl 1028.46104 MR 1974686

[5] M. Dadarlat and E. Guentner, Uniform embeddability of relatively hyperbolic groups. Preprint 2005. arXiv:math.GR/0501495

[6] C. Druţu and M. Sapir, Tree-graded spaces and asymptotic cones of groups. Topology 44 (2005), 959-1058. Zbl 1101.20025 MR 2153979

[7] B. Farb, Relatively hyperbolic groups. Geom. Funct. Anal. 8 (1998), 810-840. Zbl 0985.20027 MR 1650094

[8] M. Gromov, Hyperbolic groups. In Essays in group theory, Math. Sci. Res. Inst. Publ. 8, Springer-Verlag, New York 1987, 75-263. Zbl 0634.20015 MR 0919829

[9] M. Gromov, Geometric group theory (Sussex, 1991), vol. 2: Asymptotic invariants of infinite groups. London Math. Soc. Lecture Note Ser. 182, Cambridge University Press, Cambridge 1993. Zbl 0841.20039 MR 1253544

[10] D. Groves and J. F. Manning, Dehn filling in relatively hyperbolic groups. Preprint 2006. arXiv:math.GR/0601311

[11] G. Higman, B. H. Neumann, and H. Neumann, Embedding theorems for groups. J. London Math. Soc. 24 (1949), 247-254. Zbl 0034.30101 MR 0032641

[12] R. C. Lyndon and P. E. Schupp, Combinatorial group theory. Classics in Mathematics, Springer-Verlag, Berlin 2001. Zbl 0997.20037 MR 1812024

[13] I. Mineyev and G. Yu, The Baum-Connes conjecture for hyperbolic groups. Invent. Math. 149 (2002), 97-122. Zbl 1038.20030 MR 1914618 
[14] B. B. Newman, Some results on one-relator groups. Bull. Amer. Math. Soc. 74 (1968), 568-571. Zbl 0174.04603 MR 0222152

[15] A. Yu. Ol'shanskiı̌, On residualing homomorphisms and $G$-subgroups of hyperbolic groups. Internat. J. Algebra Comput. 3 (1993), 365-409. Zbl 0830.20053 MR 1250244

[16] D. V. Osin, Small cancellations over relatively hyperbolic groups and embedding theorems. Preprint 2004. arXiv:math.GR/0411039

[17] D. V. Osin, Asymptotic dimension of relatively hyperbolic groups. Internat. Math. Res. Notices 2005 (2005), 2143-2161. Zbl 1089.20028 MR 2181790

[18] D. V. Osin, Peripheral fillings of relatively hyperbolic groups. Invent. Math. 167 (2007), 295-326. Zbl 05133236 MR 2270456

[19] D. V. Osin, Relatively hyperbolic groups: intrinsic geometry, algebraic properties, and algorithmic problems. Mem. Amer. Math. Soc. 179 (2006). Zbl 1093.20025 MR 2182268

[20] N. Ozawa, Boundary amenability of relatively hyperbolic groups. Topology Appl. 153 (2006), 2624-2630. Zbl 05053848 MR 2243738

[21] J. Roe, Lectures on coarse geometry. Univ. Lecture Ser. 31, Amer. Math. Soc., Providence, RI, 2003. Zbl 1042.53027 MR 2007488

[22] J. Roe, Hyperbolic groups have finite asymptotic dimension. Proc. Amer. Math. Soc. 133 (2005), 2489-2490. Zbl 1070.20051 MR 2146189

[23] J. Rosenberg, $C^{*}$-algebras, positive scalar curvature, and the Novikov conjecture. Inst. Hautes Études Sci. Publ. Math. 58 (1983), 197-212. Zbl 0526.53044 MR 720934

[24] G. Skandalis, J. L. Tu, and G. Yu, The coarse Baum-Connes conjecture and groupoids. Topology 41 (2002), 807-834. Zbl 1033.19003 MR 1905840

[25] A. Yaman, A topological characterisation of relatively hyperbolic groups. J. Reine Angew. Math. 566 (2004), 41-89. Zbl 1043.20020 MR 2039323

[26] G. Yu, The coarse Baum-Connes conjecture for spaces which admit a uniform embedding into Hilbert space. Invent. Math. 139 (2000), 201-240. Zbl 0956.19004 MR 1728880

[27] G. Yu, Higher index theory of elliptic operators and geometry of groups. In Proc. Internat. Congr. Math. (Madrid, 2006), Vol. II, EMS Publ. House, Zurich 2006, 1623-1639. Zbl 05057463

Received June 5, 2006; revised October 25, 2006

D. Groves, Mathematics 253-37, California Institute of Technology, Pasadena, CA 91125, U.S.A.

E-mail: groves@caltech.edu

J. F. Manning, Department of Mathematics, University of Buffalo, Buffalo, N.Y., 14260-2900, U.S.A

E-mail: j399m@buffalo.edu 